\title{
Cool and hot executive functions in medication-naive attention deficit hyperactivity disorder children
}

\author{
B.-R. Yang ${ }^{1,2,3}$, R. C. K. Chan ${ }^{1,2 *}$, N. Gracia ${ }^{4}$, X.-Y. Cao ${ }^{1,5}$, X.-B. Zou ${ }^{6}$, J. Jing ${ }^{7}$, J.-N. Mai ${ }^{8}$ J. Li $^{9}$ \\ and D. Shum ${ }^{4}$ \\ ${ }^{1}$ Neuropsychology and Applied Cognitive Neuroscience Laboratory, Key Laboratory of Mental Health, Institute of Psychology, Chinese Academy of \\ Sciences, Beijing, People's Republic of China \\ ${ }^{2}$ Neuropsychology and Applied Cognitive Neuroscience Laboratory, Department of Psychology, Sun Yat-Sen University, Guangzhou, \\ People's Republic of China \\ ${ }^{3}$ Shenzhen Children's Hospital, Shenzhen, People's Republic of China \\ ${ }^{4}$ School of Psychology and Griffith Health Institute, Griffith University, Brisbane, Australia \\ ${ }^{5}$ Graduate School, Chinese Academy of Sciences, Beijing, People's Republic of China \\ ${ }^{6}$ Child Developmental Behavior Center, the Third Affiliated Hospital, Sun Yat-Sen University, Guangzhou, People's Republic of China \\ ${ }^{7}$ School of Public Health, Sun Yat-Sen University, Guangzhou, People's Republic of China \\ ${ }^{8}$ Guangzhou Children's Hospital, Guangzhou, People's Republic of China \\ ${ }^{9}$ Maternal and Child Health Hospital, Zhuhai, People's Republic of China
}

Background. This study aimed to compare 'cool' [working memory (WM) and response inhibition] and 'hot' (delay aversion) executive functions (EFs) in children with and without attention deficit hyperactivity disorder (ADHD).

Method. A total of 100 ADHD children (45 with family history of ADHD and 55 with no family history) and 100 healthy controls, all medication free, were tested on tasks related to the 'hot' (i.e. two choice-delay tasks) and 'cool' domains of EF (i.e. Digits backward, Corsi Block Task backward, Go/No-Go Task, Stop-Signal Task, and the Stroop).

Results. Compared with the controls, children with ADHD were found to perform significantly worse on one or more measures of response inhibition, WM, and delay aversion after controlling for co-morbidities and estimated IQ. In addition, comparisons between ADHD children with family history of ADHD and those with no family history found significant differences on measures of response inhibition and WM but not delay aversion. These results are largely supported by results of two logistic regressions.

Conclusions. ADHD was found to be associated with deficits on both cool and hot EFs. There is also evidence to suggest that cool EFs impairment is related to a family history of ADHD. Findings of this study have helped to elucidate the nature and extent of EF deficits in children with ADHD.

Received 21 April 2010; Revised 23 April 2011; Accepted 30 April 2011; First published online 1 June 2011

Key words: Attention deficit hyperactivity disorder, delay aversion, executive functions, response inhibition, working memory.

\section{Introduction}

Executive functions (EFs) are a collection of higherorder capacities that enable flexible goal-directed behavior (Welsh \& Pennington, 1988). They have been linked to functioning of the prefrontal cortex and through it to parietal, temporal and limbic lobe structures and the striatum (Roth \& Saykin, 2004). A primary weakness in EFs has been proposed to underlie the symptoms of attention deficit hyperactivity disorder (ADHD) because ADHD children

* Address for correspondence: R. C. K. Chan, Ph.D., Institute of Psychology, Chinese Academy of Sciences, 4A Datun Road, Beijing 100101, People's Republic of China.

(Email : rckchan@psych.ac.cn) [R. C. K. Chan]

(Email : d.shum@griffith.edu.au) [D. Shum] appear to lack attentional and strategic flexibility, often fail to monitor behavior effectively, and tend to display poor planning and working memory (WM) (Barkley, 1997; Castellanos \& Tannock, 2002).

Research with children with ADHD has focused on the more purely cognitive or ' $\mathrm{Cool}^{\prime} \mathrm{EFs}$ such as response inhibition and WM, associated with the dorsolateral prefrontal cortex, because some authors view ADHD as primarily a cool EF disorder (Zelazo \& Müller, 2002). Recently, 'hot' EFs, those involving emotional and motivational processes, such as measured by affective decision-making tasks (e.g. delayed aversion) and thought to be dependent on the ventromedial prefrontal cortex, have received more attention (Kerr \& Zelazo, 2004). However, to date, only one study (Solanto et al. 2001) has examined both cool and 
hot EF differences between ADHD children and normal controls.

\section{Cool EFs}

The ability to inhibit a pre-potent response in favor of another response is one of the most studied EFs in ADHD (Castellanos \& Tannock, 2002). Barkley (1997) posited that response inhibition is the primary deficit in ADHD and this in turn disrupts other EFs. Studies have consistently found that children with ADHD display slower inhibition of pre-potent responses than controls (Oosterlaan et al. 1998; Nigg, 2000), a finding that cannot be accounted for by the presence of comorbidities in ADHD children (Barkley et al. 2001).

Evidence for Barkley's hypothesis comes from research using Go/No-Go tasks and the Stop-Signal paradigm. A deficit in response inhibition is supported by a meta-analysis of 17 studies that used the Stop-Signal paradigm, which yielded a moderate overall effect size [Cohen's $d$ for Stop-Signal Reaction Time $(S S R T)=0.58$; Lijffijt et al. 2005]. Despite these findings, there are still doubts as to whether response inhibition is the primary deficit in ADHD (Castellanos et al. 2006), particularly in view of the results of a study of stimulant-naive boys with ADHD. In that study (Rhodes et al. 2005), reaction time (RT) on Go/No-Go tasks for these boys was similar to that for matched controls, suggesting that there is not a general deficit in inhibition in EF tasks in ADHD.

Research on response inhibition deficits may be useful in delineating genes related to risk for a neuropsychologically distinct subtype of ADHD (Schachar et al. 2005). Crosbie \& Schachar (2001), for example, found that children who displayed poor stop-task inhibition were significantly more likely to have a first-degree relative with ADHD than those children who exhibited good inhibition.

WM is a second EF studied extensively in ADHD and one that has been proposed as a potential neurocognitive endophenotype for the disorder (Castellanos \& Tannock, 2002). WM refers to the online storage and processing of information over short periods of time (Smith \& Jonides, 1999). In their 2005 meta-analysis, Martinussen et al. (2005) identified 26 studies and found deficits in both verbal and spatial WM for participants with ADHD that were independent of comorbidity. They also found that spatial storage and spatial central executive tasks to have larger effect sizes than their verbal counterparts.

Despite these results, findings of WM deficits in ADHD remain inconclusive and this has led Pennington \& Ozonoff (1996) to question the validity of a WM deficit associated with ADHD. Furthermore, it remains unclear whether response inhibition and
WM impairments represent distinct ADHD deficits or whether both are manifestations of a common underlying dysfunction (Castellanos \& Tannock, 2002).

\section{Hot EFs}

One view of impulsive behavior in ADHD is that it is not a result of an inability to inhibit a response, but of a rational preference to avoid delay, which an individual with ADHD finds aversive (Sonuga-Barke et al. 1992). When allowed to choose between smallersooner rewards and larger long-term rewards, children with ADHD choose the former more often than controls (Marco et al. 2009; Scheres et al. 2010). Also, compared with controls, adults with ADHD showed hypo-responsiveness of the ventral-striatal reward system for both immediate and delayed rewards, suggesting a diminished neural processing of reward (Plichta et al. 2010). In addition, these adults showed hyper-activation in the dorsal caudate nucleus and amygdale, suggesting a preference for immediate rather than delayed rewards even though the intrinsic value of the latter was smaller. Given the possibility that impulsive behavior in ADHD children might be due to their tendency for delay aversion, it is important to clarify this issue by including both cool and hot EFs measures in the one study.

According to Bitsakou et al. (2009), delay aversion may have a family etiology (biological and social). A twin study by Kuntsi et al. (2001) found low-level heritability and significant effects for shared environment, consistent with a family etiology. Other studies, however, have failed to find familial influences (Andreou et al. 2007; Bidwell et al. 2007).

\section{Rationale and aims of study}

In the one study to date that compared both cool and hot EFs in ADHD children and controls, Solanto et al. (2001) tested participants on both delay aversion and response inhibition tasks. They reported deficits for ADHD children on both tasks, but the differences were more pronounced on delay aversion.

The present study sought to extend the comparison of hot and cool EF differences between children with and without ADHD by including a more extensive battery of response inhibition and WM tasks, as well as SST and delay version tasks used by Solanto et al. (2001). Because previous studies of EFs and ADHD had shown small effects, a large sample size was employed and an attempt was made to eliminate the possible confounding in some previous studies of ADHD status with other disorders by statistically controlling for co-morbidities. Importantly, the present study was able to include only medication-naive 
ADHD children in the study and so control for possible confounding of comparisons arising from drug effects. Given that results of some previous studies have provided evidence to suggest that EF impairments might have a family etiology, a secondary aim of the present study was to explore this possibility in our sample.

It was hypothesized that (1) compared with controls, children with ADHD would show significantly poorer performance on measures of response inhibition, WM and delay aversion; (2) children with ADHD and a family history of disorder would perform significantly more poorly on measures of response inhibition, WM and delay aversion than children with ADHD without a family history.

\section{Method}

\section{Participants}

Participants in the study comprised 100 children with ADHD and 100 healthy controls. There were 90 boys and 10 girls in the ADHD group (mean age 8.42 years, S.D. $=1.59$ years) with a mean estimated IQ based on four subtests from the Chinese Revised Wechsler Intelligence Scale for Children (C-WISC; Gong \& Cai, 1993) of 99.32 (S.D. =12.1). There were 89 boys and 11 girls in the control group (mean age 8.49 years, S.D. $=1.58$ years) with a mean estimated IQ of 106.82 (S.D.=11.12). Children with ADHD were recruited from consecutive referrals to three child behavioral clinics that serve large urban populations in the Guangdong Province, China. The controls were recruited from a primary school in the same province. All participants had to meet the following criteria: (1) age between 6 to 12 years; (2) estimated IQ > 75; (3) normal auditory and normal or corrected to normal visual acuity; (4) no nervous system diseases and other medical problems which would make an impact on mental functions; (5) no developmental language problems; (6) having never received psychoactive medications; and (7) for participants in the control group not diagnosed with autism or other mental disorders.

Inclusion in the ADHD group required a diagnosis of ADHD based on semi-structured parent and child interviews with a consultant pediatrician. Additional inclusion criteria for this group were: (1) parent and/or teacher complaints of inattention, poor impulse control, and over-activity; (2) at least six of the 18 inattention or hyperactivity-impulsive symptoms on the ADHD checklist or scores by informant at or above the clinical cut-off on the Conners' Rating Scale (CRS-48; Conners, 1989) or the Child Behavioral Checklist (CBCL; Achenbach, 1978); (3) met all the
Diagnostic and Statistical Manual of Mental Disorders, Fourth Edition (DSM-IV; APA, 1994) criteria for ADHD during the clinical interview; and (4) never received psychoactive medications or other psychological therapies.

Among the children with ADHD, 45 had a family history of ADHD and 55 had no family history of ADHD. Given the exploratory nature of this research question, data on family history of ADHD were collected from parents using two questions: whether either parent or any of either parent's siblings had displayed symptoms of ADHD during childhood. A positive family history was recorded if one or both parents answered yes to either or both of the two questions asked.

Table 1 presents the demographic and clinical characteristics of the two ADHD groups and the controls. Among the 100 children with ADHD, 36 had a co-morbid diagnosis of some form: 22 had had co-morbid oppositional defiant disorder or conduct disorder, 11 had learning disability, and three had tic disorder.

\section{Measures}

Response inhibition was assessed using the Go/ No-Go Task (van der Meere \& Stemerdink, 1999), the Stop-Signal Task (SST; McInerney \& Kerns, 2003) and a Chinese version of the Stroop Test (CST; Lee \& Chan, 2000).

The Go/No-Go Task comprised a total of 200 stimuli, $80 \%$ being ' $G o^{\prime}$ (letter ' $\mathrm{R}$ ' displayed) and 20\% 'No-Go' trials (' $\mathrm{P}^{\prime}$ displayed). Participants were presented with $\mathrm{a}^{\prime}+{ }^{\prime}$ at the centre of the computer screen for $400 \mathrm{~ms}$, followed by either a Go or No-Go stimulus for $200 \mathrm{~ms}$. This was followed by an interstimulus interval that randomly varied between 600 to $1200 \mathrm{~ms}$. Measures obtained for this task were number of commission errors, and mean RT.

The SST (Logan et al. 1997) comprised two trial blocks with 48 trials in each: 32 response signal trials without stop signals and 16 with stop signals. The response signal stimulus was either an ' $\mathrm{X}$ ' or ' $\mathrm{O}$ ', each with an equal chance of being presented in a block. In the SST, children were given two concurrent tasks, a Go task requiring them to discriminate between the two response signal stimuli (by pushing their corresponding buttons), and a Stop task requiring them to inhibit their Go task responses when a white frame appeared around the response stimulus. Difficulty of the task was varied by adjusting the speed at which the frame appeared (increasing or decreasing by $50 \mathrm{~ms}$ steps) to ensure that the success rate of all participants was approximately 50\%. The measure obtained for this task was the mean SSRT. 
Table 1. Demographic and clinical characteristics of children with ADHD and controls

\begin{tabular}{|c|c|c|c|c|c|c|c|}
\hline & ADHD & Controls & $F$ & $p$ & $\mathrm{ADHD}+$ & ADHD - & $\begin{array}{l}\mathrm{ADHD}+v \\
\mathrm{ADHD}-: p\end{array}$ \\
\hline Age, years & $8.418(0.159)$ & $8.488(0.158)$ & 0.097 & 0.755 & $8.391(0.243)$ & $8.440(0.213)$ & 0.880 \\
\hline Grade & $2.450(0.145)$ & $2.450(0.145)$ & 0.000 & 1.000 & $2.470(0.217)$ & $2.440(0.196)$ & 0.918 \\
\hline Estimated IQ & $98.520(1.141)$ & $106.822(1.113)$ & 27.155 & $<0.001$ & $96.909(1.729)$ & $99.833(1.507)$ & 0.204 \\
\hline $\begin{array}{l}\text { Conners' Parent Rating Scale } \\
\text { (hyperactivity index) }\end{array}$ & $1.437(0.064)$ & $0.346(0.043)$ & 165.521 & $<0.001$ & $1.447(0.107)$ & $1.426(0.072)$ & 0.869 \\
\hline $\begin{array}{l}\text { Conners' Teacher Rating Scale } \\
\text { (hyperactivity index) }\end{array}$ & $1.515(0.085)$ & $0.307(0.043)$ & 147.097 & $<0.001$ & $1.548(0.120)$ & $1.450(0.121)$ & 0.433 \\
\hline Child Behavior Checklist total & $59.080(2.812)$ & $13.390(1.574)$ & 166.049 & $<0.001$ & $62.280(4.883)$ & $56.200(3.029)$ & 0.283 \\
\hline
\end{tabular}

Data are given as mean (standard error).

ADHD, Attention deficit hyperactivity disorder; ADHD +, ADHD children with a family history of the disorder ; ADHD - , ADHD children without a history of the disorder.

The CST was based on the Stroop Test-Victoria Version (Regard, 1981), except that the common words (part W) and color words (part C) were replaced by Chinese characters. Common words are written in colored ink but have no semantic connection to the color, whereas color words are semantically related to the color but are written in an unrelated colored ink. The color dots (part D) of the Victorian version remained the same. The participants were asked to read the color of dots or words, not the words themselves. The measure obtained for this test was the error for the interference trial.

WM was assessed verbally and visually. The Digits backward condition of the Digit Span subtest of the C-WISC (Gong \& Cai, 1993) was used for assessing verbal WM. The total number of correctly recalled trials was the measure taken. The backward condition of the Corsi Blocks Task (CBT; Vandierendonck et al. 2004) was used for assessing visual WM. Verbal and visuospatial $n$-back tasks were also administered to the participants as measures of WM. However, measures of these tasks were not used in the data analyses because there were too many missing data. For the CBT, nine identical cubes were positioned irregularly on a board. During testing, the examiner pointed to a series of blocks at a rate of one per s with children required to point to the blocks in the reverse order presented. The total number of correct trials was the measure taken.

Delay aversion was assessed using two versions of the Choice-Delay Task (CDT). CDT-1 was modified from Solanto et al. (2001). Children were told that they would take part in a game and the higher their score, the better. Using a computer mouse they were asked to choose between two circles (one is associated with a 1-point reward and the other a 2-point reward) presented in the centre of the screen. After selecting one of the two circles, there was a delay of either $2 \mathrm{~s}$ or $30 \mathrm{~s}$ before the number of points was posted on the screen. Before the task, children were told that: (a) there was no time limit; $(b)$ there would be 10 'tries' to earn points; and (c) one chip would be placed on a grid for each try so that they could keep track of how many tries were left. The total number of 2-point rewards chosen and the RT for each choice were recorded as measures of delay aversion.

To improve the ecological validity of delay aversion, this study also included CDT-2, as suggested by van Goozen et al. (2004). In this task children were asked to make a choice between two circles, one marked ' $40 \%$ ' which had a $40 \%$ chance of receiving 1 point immediately if chosen, and the other marked ' $80 \%$ ' which had an $80 \%$ chance of receiving 1 point in $12 \mathrm{~s}$. In total, 20 test trials were administered, with no time limit set for the task. The total number of choices for the circles marked ' $80 \%$ ' and the choice RT were recorded as measures of delay aversion.

\section{Procedure}

The Sun Yat-Sen University human research ethics committee granted approval for the project and written consent was provided by each child's guardian. Children were tested in a quiet room either in a clinic or at their school and administered the battery of neurocognitive tests in a fixed order designed to minimize boredom.

\section{Statistical analysis}

Demographic and clinical characteristics of groups were compared using $\chi^{2}$ analysis for categorical variables and analysis of variance for continuous scores. Differences between means for groups were tested using univariate analysis of covariance. Comparisons involved the total patient group with the control 
Table 2. Comparisons of EF performance based on patient status and family history with co-morbidities and IQ controlled

\begin{tabular}{|c|c|c|c|c|c|c|c|}
\hline & ADHD & Controls & $F$ & $p$ & $\mathrm{ADHD}+$ & ADHD - & $\begin{array}{l}\mathrm{ADHD}+v . \\
\mathrm{ADHD}-: p\end{array}$ \\
\hline \multicolumn{8}{|l|}{ Response inhibition } \\
\hline $\begin{array}{l}\text { Go/No-Go } \\
\text { commission error }\end{array}$ & $13.489(0.556)$ & $12.083(0.518)$ & 2.969 & 0.087 & $14.115(0.789)$ & 12.999 (0.717) & 0.276 \\
\hline Go/No-Go RT & $451.855(13.672)$ & $448.378(12.745)$ & 0.030 & 0.863 & $463.790(19.653)$ & 442.400 (17.678) & 0.399 \\
\hline $\begin{array}{l}\text { CST interference } \\
\text { error }\end{array}$ & $2.100(0.208)$ & $1.220(0.204)$ & 7.898 & 0.005 & $2.430(0.307)$ & $1.865(0.263)$ & 0.146 \\
\hline SST SSRT & $307.500(14.911)$ & $271.593(13.474)$ & 2.671 & 0.104 & $348.580(20.518)$ & 274.500 (18.609) & 0.005 \\
\hline \multicolumn{8}{|l|}{ Working memory } \\
\hline Digits backward & $3.797(0.229)$ & $6.177(0.225)$ & 47.702 & $<0.001$ & $3.482(0.333)$ & $4.032(0.292)$ & 0.196 \\
\hline CBT backward & $6.812(0.186)$ & $8.475(0.184)$ & 35.149 & $<0.001$ & $5.704(0.251)$ & $7.621(0.217)$ & $<0.001$ \\
\hline \multicolumn{8}{|l|}{ Delay aversion } \\
\hline CDT-1 total & $9.768(0.513)$ & $9.949(0.497)$ & 0.056 & 0.814 & $9.755(0.768)$ & $9.777(0.643)$ & 0.982 \\
\hline CDT-1 RT & 1592.477 (185.200) & 1519.562 (179.543) & 0.069 & 0.793 & $1662.500(277.280)$ & 1545.000 & )) 0.734 \\
\hline CDT-2 total & $4.487(0.266)$ & $5.476(4.487)$ & 6.138 & 0.014 & $4.191(0.396)$ & $4.191(0.331)$ & 0.138 \\
\hline CDT-2 RT & $1815.412(133.850)$ & $1403.415(129.755)$ & 4.208 & 0.042 & $1881.300(200.350)$ & $1771.000(167.700)$ & 0.658 \\
\hline
\end{tabular}

Data are given as mean (standard error).

$\mathrm{EF}$, Executive function; ADHD, attention deficit hyperactivity disorder; ADHD +, ADHD children with a family history of the disorder; ADHD - , ADHD children without a history of the disorder; RT, reaction time; CST, Chinese version of the Stroop Test; SST, Stop-Signal Task; SSRT, Stop-Signal reaction time; CBT, Corsi Blocks Task; CDT, Choice-Delay Task.

group (to test for the effect of patient status) and then the positive family history patient group with the negative family history patient group (to test for the effect of family history) and each with the control group. Co-morbidity and IQ were used as covariates and comparisons were made on the means following adjustment for the covariates.

The relative contribution of EF variables to the prediction of patient status and family history was assessed using stepwise logistic regressions. Comorbidity and IQ were entered at the first step to adjust for differences in these and then WM, response inhibition, and delay aversion measures were entered sequentially in blocks. Collinearity was checked in terms of bivariate and multivariate correlations in the predictor set and residuals were checked using standard regression diagnostics for poor model fit and points of undue influence. Departures were minor. Where tests of significance were made, an $\alpha$ of $p<0.05$ (two tailed) was employed.

\section{Results}

\section{Demographic and clinical characteristics}

As can be seen from Table 1, the ADHD children and controls were not significantly different on age and grade. The estimated IQ of the former group was found to be significantly lower than that of the latter group but the scores of the former group on the
Conners' Rating Scale (both parent and teacher) and the CBCL were found be significantly higher than those of the latter group. In addition, the two groups of participants were not significantly different in gender and handedness $(p>0.05)$. The two ADHD groups were not found to be significantly different on any of the demographic and clinical characteristics.

\section{Hot and cool EFs}

Table 2 presents a summary of comparisons on each of the EF measures for the effects of patient status and family history. Inspection of the table shows the following measures discriminated the patient and control groups at the nominated $\alpha$ level: the two WM measures (Digits backward and CBT backward), one response inhibition measure (Stroop interference error score) and two delayed aversion measures (CDT-2 number and CDT-2 RT). For family history, only CBT backward and SST SSRT measures significantly discriminated the patient group with a positive family history of ADHD from the patient group with no family history of ADHD.

Stepwise logistic regression was used to predict patient status and then family history from the following blocks of predictors: WM (Digits backward and CBT backward), response inhibition (Stroop interference error score, Go/No-Go RT, Go/No-Go commission error, and SST SSRT) and delayed aversion (CDT-1 total, CDT-1 RT, CDT-2 total and CDT-2 
Table 3. Regression coefficients for the prediction of patient status

\begin{tabular}{lrrr}
\hline Variable & $\beta$ (s.E.) & $p$ & Odds ratio (95\% CI) \\
\hline Response inhibition & & & $0.981(0.872-1.103)$ \\
Go/No-Go commission error & $-0.019(0.060)$ & 0.746 & $0.993(0.987-0.999)$ \\
Go/No-Go RT & $-0.007(0.003)$ & 0.015 & $1.235(0.925-1.650)$ \\
CST interference error & $0.211(0.148)$ & 0.152 & $0.998(0.994-1.003)$ \\
SST SSRT & $-0.002(0.002)$ & 0.454 & $0.482(0.344-0.675)$ \\
Working memory & & & $0.431(0.283-0.656)$ \\
Digits backward & $-0.730(0.172)$ & 0.000 & $0.986(0.865-1.124)$ \\
CBT backward & $-0.842(0.214)$ & & $1.000(0.999-1.000)$ \\
Delay aversion & & 0.835 & $0.665(0.504-0.877)$ \\
CDT-1 total & $-0.014(0.067)$ & 0.353 & $1.000(1.000-1.001)$ \\
CDT-1 RT & $0.000(0.000)$ & 0.004 & 0.205 \\
CDT-2 total & $-0.408(0.141)$ & 0.000 & \\
CDT-2 RT & $0.000(0.000)$ & $15.639(4.213)$ & \\
Constant & & & \\
\hline
\end{tabular}

S.E., Standard error; CI, confidence interval; RT, reaction time; CST, Chinese version of the Stroop Test; SST, Stop-Signal Task ; SSRT, Stop-Signal reaction time; CBT, Corsi Blocks Task; CDT, Choice-Delay Task.

RT). This set of predictors covered the three domains of EF of interest and included the measures found to separate the groups in the univariate analyses. The covariates of co-morbidity and IQ were entered in the first block.

The logistic regression with patient status $(0=$ control, $1=$ patient) as the criterion indicated a reduction in the -2LL (log-likelihood) with the addition of the two covariates at the first step $[\Delta=61.330$, degrees of freedom $(\mathrm{df})=2, p<0.001]$ and with the WM measures at the second step $(\Delta=53.703, \mathrm{df}=2$, $p<0.001)$. The reduction at the third step, the introduction of the response inhibition measures, was not statistically significant $(\Delta=6.671, \mathrm{df}=4, p=0.154)$, but the reduction at the fourth step with the introduction of the hot EF measures was $(\Delta=11.363, \mathrm{df}=4$, $p=0.023)$. Model fit for the final model was satisfactory (Hosmer \& Lemeshow $\chi^{2}=10.127, \mathrm{df}=8$, $p=0.256$, Cox \& Snell $R^{2}=0.539$, Neagelkerke $R^{2}=$ $0.720)$. However, the best fit in terms of the Hosmer and Lemeshow $\chi^{2}$ was obtained with the covariates and the WM measures in the model $\left(\chi^{2}=1.937, p=\right.$ 0.983, Cox \& Snell $R^{2}=0.488$, Nagelkerke $R^{2}=0.652$ ).

The $\beta$ weights and odds ratios for each of the predictors in the final model predicting patient status are shown in Table 3. The $\beta$ weights for Digits backward, CBT backward, Go/No-Go RT, and CDT-2 total were statistically significant and the confidence intervals on the odds ratios for these variables do not include 1 , although in the case of Go/No-Go RT the upper bound is close to 1 . The negative signs and the odds ratios less than 1 indicate that high scores on these variables increase the likelihood of being in the control rather than the patient group.
The logistic regression with family history $(0=$ ADHD-, $1=\mathrm{ADHD}+$ ) as the criterion showed a statistically significant reduction in -2LL with the introduction of WM measures over the covariates alone in the model $(\Delta=44.306, \mathrm{df}=2, p<0.001)$ but the reductions for no other steps were statistically significant. The final model with the covariates and all measures was a marginally better fit (Hosmer \& Lemeshow $\chi^{2}=5.709, \mathrm{df}=8, p=0.680$, Cox \& Snell $R^{2}=0.468$, Nagelkerke $\left.R^{2}=0.626\right)$ than the model with only the covariates and the WM measures (Hosmer \& Lemeshow $\chi^{2}=7.024, \mathrm{df}=8, p=0.534$, Cox \& Snell $R^{2}=0.438$, Nagelkerke $R^{2}=0.586$ ).

The $\beta$ weights and odds ratios for each of the predictors in the final model predicting family history are shown in Table 4 . Only the $\beta$ weight for CBT backward was statistically significant and the confidence intervals for the odds ratio in this case do not include 1 . The negative sign for the $\beta$ weight and the odds ratio less than 1 indicate that high scores on the block recall test mean that the participant is less likely to belong to the group with the positive family history of ADHD.

\section{Discussion}

This study compared a relatively large group of medication-naive children with ADHD with healthy controls using a number of cool and hot EF tasks. It also compared ADHD children with and without a family history of the disorder on these measures. For cool EFs, results revealed response inhibition and WM deficits in children with ADHD. Among the response inhibition tasks, children with ADHD were found to perform significantly worse than the controls on the 
Table 4. Regression coefficients for the prediction of family history

\begin{tabular}{lcrl}
\hline Variable & $\beta$ (s.E.) & $p$ & Odds ratio (95\% CI) \\
\hline Response inhibition & & & \\
Go/No-Go commission error & $0.037(0.0114)$ & 0.749 & $1.037(0.829-1.298)$ \\
Go/No-Go RT & $0.000(0.004)$ & 0.990 & $1.000(0.992-1.008)$ \\
CST interference error & $0.157(0.192)$ & 0.411 & $1.171(0.803-1.708)$ \\
SST SSRT & $-0.004(0.003)$ & 0.159 & $0.996(0.990-1.002)$ \\
Working memory & & & $0.883(0.549-1.421)$ \\
Digits backward & $-0.125(0.243)$ & 0.608 & $0.177(0.072-0.435)$ \\
CBT backward & $-1.732(0.459)$ & $<0.001$ & $0.939(0.774-1.140)$ \\
Delay aversion & & & $1.000(0.999-1.001)$ \\
CDT-1 total & $-0.062(0.099)$ & 0.527 & $1.138(0.837-1.548)$ \\
CDT-1 RT & $0.000(0.000)$ & 0.805 & $1.000(0.999-1.001)$ \\
CDT-2 total & $0.129(0.157)$ & 0.410 & 0.969 \\
CDT-2 RT & $0.000(0.000)$ & 0.026 & \\
Constant & $12.790(5.728)$ & & \\
\hline
\end{tabular}

S.E., Standard error; CI, confidence interval; RT, reaction time; CST, Chinese version of the Stroop Test; SST, Stop-Signal Task ; SSRT, Stop-Signal reaction time; CBT, Corsi Blocks Task; CDT, Choice-Delay Task.

Stroop. In addition, the Go/No-Go task was found to be a significant predictor in discriminating the ADHD and control groups. The only task that was not found to be impaired in the ADHD children was the StopSignal paradigm. This is surprising given that Lijffijt et al. (2005) reported a moderate effect for this task in their meta-analysis of 17 studies. Given the size of our sample, this difference in results is not likely to be due to insufficient power. Two probable reasons are: only medication-naive participants were included in the current study and the study statistically controlled comorbidities in the between-group analyses. More research is needed to clarify this issue. As hypothesized, evidence for familial effects on response inhibition was also found. This was evident from the poorer performance on those with a family history of ADHD compared with those without a family history on the SST SSRT. This finding is similar to that reported by Crosbie \& Schachar (2001) who found that ADHD children who showed poorer response inhibition (as measured by SST SSRT) were more likely to have a first-degree relative with ADHD than ADHD children who showed good response inhibition. It lends support to suggestions that a response inhibition deficit is affected by genetic factors.

Children with ADHD were also found to be impaired on the other type of cool EF, namely, WM. Specifically, differences were found on Digits backward and CBT backward, with the ADHD group showing significantly poorer performance on these measures compared with the controls. This finding is further supported by the results of a logistic regression which found these two measures to significantly discriminate the ADHD and control groups. The finding is consistent with the conclusion of the metaanalysis reported by Martinussen et al. (2005). Similar to the findings for response inhibition, results of the WM analyses also lend support to the hypothesis that family predisposition may be involved in ADHD. Specifically, comparisons revealed poorer performance on the CBT backward for those with a family history of ADHD compared with those without such a history. This finding is supported by the results of the logistic regression analysis that showed that CBT backward significantly discriminated ADHD children with and without a family history of the disorder.

Overall, among the results of cool EF measures, those associated with WM seem to be more robust and consistent than those associated with response inhibition. Although this points to the possibility that WM impairment might be a primary deficit, more research is needed before a firm conclusion can be drawn.

For the hot EFs of delay aversion, the results of this study found significant differences on the two measures of CDT- 2 but not the two measures of CDT- 1 between the ADHD and control groups. Specifically, children with ADHD were found to prefer stimuli that have a short delay in providing outcome or feedback even though these stimuli might be associated with a lower score. This finding is consistent with the results of the logistic regression that found that total score on CDT-2 significantly discriminated the ADHD children and controls. Unlike Solanto et al. (2001), the current study did not find ADHD children to be impaired on measures of CDT-1. This difference in results is difficult to explain because the two studies are similar in 
many ways: mean age of participants, ADHD children were medication naive at the time of the study, and effects of co-morbidities taken into consideration during data analyses. One probable reason for the difference could be the smaller number of CDT- 1 trials included in this study than the Solanto et al. (2001) study. So far, the CDT-2 has rarely been used in studies of children with ADHD, with the exception of van Goozen et al. (2004). As mentioned, the CDT-2 is more ecologically valid and as such it might be a more sensitive measure of delay aversion for participants in this study. Measures of hot EFs were not found to differ significantly between ADHD children with and without a family history of the disorder. This is similar to the findings reported by Andreou et al. (2007) and Bidwell et al. (2007). Thus, unlike response inhibition and WM, the role of family disposition of this type of EF is not strong.

Overall, results of this study indicate that ADHD children are impaired on both cool and hot EFs. Although impairments of cool EFs such as response inhibition and WM in these children have been found in previous studies (e.g. Nigg, 2000; Castellanos \& Tannock, 2002), the finding that these children are also impaired on hot EFs such as delay aversion is much needed evidence for this research area because few studies have examined both hot and cool EFs together. Together these results suggest that children with ADHD are impaired in the ability to inhibit a prepotent response, the ability to actively process information in short-term storage, and the ability to delay a more advantageous response. In addition, to the extent that different areas of the prefrontal cortex are involved in cool and hot EFs, these present results suggest that ADHD children may suffer from irregularities in the dorsolateral and ventromedial prefrontal areas of the brain.

By including a relatively large group of medicationnaive ADHD children in our study and by controlling for co-morbidities, we have also avoided the confounding effects of medication history and provided a more rigorous evaluation of EF deficits in these children. By comparing ADHD children with and without a family history on cool and hot EF measures and by finding significant results for two types of cool EF (WM and response inhibition), findings of this study provide initial support for a genetic influence on these EFs. However, it should be noted that the family history data of this study were collected by questioning parents of the children with ADHD on the presence of ADHD symptoms during both their and their siblings' childhood. Because no formal diagnostic testing or interview was conducted on other family members, it is difficult to rule out the impact of under- or over-reporting of childhood symptoms.
Nonetheless, given that ADHD symptoms are easily observed by parents, any bias might be minimal, particularly in view of the fact that the incidence of ADHD with a family history of the disorder in this study was similar to that reported in North American populations (Buitelaar et al. 2006).

\section{Acknowledgements}

This study was supported partially by the Research Initiation Fund of the Hundred Talents Programme (16000-3253182) from the Sun Yat-Sen University, the key Laboratory of Mental Health, Institute of Psychology, Chinese Academy of Sciences, ProjectOriented Hundred Talents Programme (O7CX031003) of the Institute of Psychology, and the Knowledge Innovation Project of the Chinese Academy of Sciences (KSCX2-YW-R-131 and KSCX2-EW-J-8). Also, R.C.K.C. received an Outstanding Young Investigator Award of the National Science Foundation China (81088001) and D.S. received grants from the Australian Academy of Science Scientific Visit to China. These funding agents had no role in the study design, collection, analysis and interpretation of the data, writing of the manuscript, or decision to submit the paper for publication. The authors thank Professor John O'Gorman for his invaluable feedback and assistance on various versions of the manuscript.

\section{Declaration of Interest}

None.

\section{References}

Achenbach TM (1978). The child behavior profile: I. Boys aged 6-11. Journal of Consulting and Clinical Psychology 46, 478-488.

Andreou P, Neale BM, Chen W, Christiansen H, Gabriels I, Heise A, Meidad S, Muller UC, Uebel H, Banaschewski T (2007). Reaction time performance in ADHD: improvement under fast-incentive condition and familial effects. Psychological Medicine 37, 1703-1715.

APA (1994). Diagnostic and Statistical Manual of Mental Disorders, 4th edn, revised. American Psychological Association: Washington, DC.

Barkley RA (1997). Behavioral inhibition, sustained attention, and executive functions: constructing a unifying theory of ADHD. Psychological Bulletin 121, 65-94.

Barkley RA, Edwards G, Laneri M, Fletcher K, Metevia L (2001). Executive functioning, temporal discounting, and sense of time in adolescents with attention deficit hyperactivity disorder (ADHD) and oppositional defiant disorder (ODD). Journal of Abnormal Child Psychology 29, 541-556.

Bidwell LC, Willcutt EG, DeFries JC, Pennington BF (2007). Testing for neuropsychological endophenotypes in siblings 
discordant for attention-deficit/hyperactivity disorder. Biological Psychiatry 62, 991-998.

Bitsakou P, Psychogiou L, Thompson M, Sonuga-Barke EJS (2009). Delay aversion in attention deficit/hyperactivity disorder: an empirical investigation of the broader phenotype. Neuropsychologia 47, 446-456.

Buitelaar J, Barton J, Danckaerts M, Friedrichs E, Gillberg C, Hazell P, Hellemans H, Johnson M, Kalverdijk L, Masi G (2006). A comparison of North American versus non-North American ADHD study populations. European Child and Adolescent Psychiatry 15, 177-181.

Castellanos FX, Sonuga-Barke EJS, Milham MP, Tannock R (2006). Characterizing cognition in ADHD: beyond executive dysfunction. Trends in Cognitive Sciences 10, $117-123$.

Castellanos FX, Tannock R (2002). Neuroscience of attention-deficit/hyperactivity disorder: the search for endophenotypes. Nature Reviews Neuroscience 3, 617-628.

Conners CK (1989). Conners' Rating Scales Manual. Multi-Health Systems: North Tonawanda, New York.

Crosbie J, Schachar R (2001). Deficient inhibition as a marker for familial ADHD. American Journal of Psychiatry 158, 1884-1890.

Gong YX, Cai TS (1993). Manual of Chinese Revised Wechsler Intelligence Scale for Children. Hunan Atlas Publishing House: Changsha.

Kerr A, Zelazo PD (2004). Development of 'hot' executive function: the children's gambling task. Brain and Cognition 55, 148-157.

Kuntsi J, Oosterlaan J, Stevenson J (2001). Psychological mechanisms in hyperactivity: I. Response inhibition deficit, working memory impairment, delay aversion, or something else? Journal of Child Psychology and Psychiatry and Allied Disciplines 42, 199-210.

Kuntsi J, Stevenson J (2001). Psychological mechanisms in hyperactivity: II. The role of genetic factors. Journal of Child Psychology and Psychiatry and Allied Disciplines 42, 211-219.

Lee TMC, Chan CCH (2000). Stroop interference in Chinese and English. Journal of Clinical and Experimental Neuropsychology 22, 465-471.

Lijffijt M, Kenemans JL, Verbaten MN, van Engeland H (2005). A meta-analytic review of stopping performance in attention-deficit/hyperactivity disorder: deficient inhibitory motor control. Journal of Abnormal Psychology 114, 216-222.

Logan GD, Schachar RJ, Tannock RT (1997). Impulsivity and inhibitory control. Psychological Science 8, 60-64.

Marco R, Miranda A, Schlotz W, Melia A, Mulligan A, Muller U, Andreou P, Butler L, Christiansen H, Gabriels I, Medad S, Albrecht B, Uebel H, Asherson P, Banaschewski T, Gill M, Kuntsi J, Mulas F, Oades R, Roeyers H, Steinhausen H-C, Rothenberger A, Faraone SV, Sonuga-Barke EJS (2009). Delay and reward choice in ADHD: an experimental test of the role of delay aversion. Neuropsychology 23, 367-380.

Martinussen R, Hayden J, Hogg-Johnson S, Tannock R (2005). A meta-analysis of working memory impairments in children with attention-deficit/hyperactivity disorder. Journal of the American Academy of Child and Adolescent Psychiatry 44, 377-384.
McInerney RJ, Kerns KA (2003). Time reproduction in children with ADHD: motivation matters. Child Neuropsychology 9, 91-108.

Nigg JT (2000). On inhibition/disinhibition in developmental psychopathology: views from cognitive and personality psychology and a working inhibition taxonomy. Psychological Bulletin 126, 220-246.

Oosterlaan J, Logan GD, Sergeant JA (1998). Response inhibition in $\mathrm{AD} / \mathrm{HD}, \mathrm{CD}$, comorbid $\mathrm{AD} / \mathrm{HD}+\mathrm{CD}$, anxious, and control children: a meta-analysis of studies with the stop task. Journal of Child Psychology and Psychiatry and Allied Disciplines 39, 411-425.

Pennington BF, Ozonoff S (1996). Executive functions and developmental psychopathology. Journal of Child Psychology and Psychiatry 37, 51-87.

Plichta MM, Vasic N, Wolf RC, Lesch KP, Brummer D, Jacob C, Fallgatter AJ, Grön G (2010). Neural hyporesponsiveness and hyperresponsiveness during immediate and delayed reward processing in adult attention-deficit/hyperactivity disorder. Biological Psychiatry 65, 7-14.

Regard M (1981). Stroop Test - Victoria Version. Neuropsychological Laboratory, University of Victoria: Victoria, BC.

Rhodes SM, Coghill DR, Matthews K (2005). Neuropsychological functioning in stimulant-naive boys with hyperkinetic disorder. Psychological Medicine 35, 1109-1120.

Roth RM, Saykin AJ (2004). Executive dysfunction in attention-deficit/hyperactivity disorder: cognitive and neuroimaging findings. Psychiatric Clinics of North America 27, 83-96.

Schachar RJ, Crosbie J, Barr CL, Ornstein TJ, Kennedy J, Malone M, Roberts W, Ickowicz A, Tannock R, Chen S (2005). Inhibition of motor responses in siblings concordant and discordant for attention deficit hyperactivity disorder. American Journal of Psychiatry 162, 1076-1082.

Scheres A, Tontsch C, Thoeny AL, Kaczkurkin A (2010). Temporal reward discounting in attention-deficit/ hyperactivity disorder: the contribution of symptom domains, reward magnitude, and session length. Biological Psychiatry 67, 641-648.

Smith EE, Jonides J (1999). Storage and executive processes in the frontal lobes. Science 283, 1657.

Solanto MV, Abikoff H, Sonuga-Barke E, Schachar R, Logan GD, Wigal T, Hechtman L, Hinshaw S, Turkel E (2001). The ecological validity of delay aversion and response inhibition as measures of impulsivity in AD/HD: a supplement to the NIMH Multimodal Treatment Study of AD/HD. Journal of Abnormal Child Psychology 29, 215-228.

Sonuga-Barke E, Taylor E, Sembi S, Smith J (1992). Hyperactivity and delay aversion III. The effect of delay on choice. Journal of Child Psychology and Psychiatry 33, 387-398.

van der Meere J, Stemerdink N (1999). The development of state regulation in normal children: an indirect comparison with children with ADHD. Developmental Neuropsychology 16, 213-225. 
van Goozen S, Cohen-Kettenis P, Snoek H, Matthys W, Swaab-Barneveld H, van Engeland H (2004). Executive functioning in children: a comparison of hospitalised ODD and ODD/ADHD children and normal controls. Journal of Child Psychology and Psychiatry 45, 284-292.

Vandierendonck A, Kemps E, Fastame MC, Szmalec A (2004). Working memory components of the Corsi blocks task. British Journal of Psychology 95, 57-79.
Welsh MC, Pennington BF (1988). Assessing frontal lobe functioniong in children: views from developmental psychology. Developmental Neuropsychology 4, 199-230.

Zelazo PD, Müller U (2002). Executive function in typical and atypical development. In Handbook of Childhood Cognitive Development (ed. U. Goswami), pp. 445-469. Blackwell: Malden, MA. 